\title{
THE PRODUCT OF COMMUTING CONICAL PROJECTIONS IS A PROJECTION
}

\author{
EDUARDO H. ZARANTONELLO
}

\begin{abstract}
The statement in the title is proved for two projections on closed convex cones in Hilbert space.
\end{abstract}

The projection on a closed convex set $K$ in a real Hilbert space $H$ is the operator $P_{K}$ assigning to each $x \in H$ the nearest point in $K$. In a recent paper devoted to the study of these operators [1] the author formulated the conjecture that the product of any two commuting projections is a projection again, namely the projection on the intersection of the corresponding convex sets $[1$, p. 325], and proved its validity for projections on finite dimensional closed convex cones [1, Theorem 5.6]. In the present article the original proof is extended to any two commuting projections on cones, eliminating the finite dimensionality requirement. In addition included is a brief proof, already implicit in our previous paper [1, Lemma 5.3], that commutativity is also necessary for the products of two such projections to be projections.

The discussion, entirely elementary, is based on the following simple facts: The variational inequalities for the problem of minimizing the distance to a closed convex set $K$-known as the Bourbaki-CheneyGoldstein inequalities-are

$$
\left\langle x-P_{K} x, P_{K} x-y\right\rangle \geqq 0, \quad \forall y \in K,
$$

and characterize $P_{K} x$ entirely, that is,

(2) $\left\{z=P_{K} x\right\} \Leftrightarrow\{\langle x-z, z-y\rangle \geqq 0, z \in K, \forall y \in K\} \quad$ [1, Lemma 1.2].

Setting $y=P_{K} x^{\prime}$ in (1) and adding the resulting inequality to that obtained by interchanging $x$ and $x^{\prime}$, one deduces

$$
\left\langle x-x^{\prime}, P_{K} x-P_{K} x^{\prime}\right\rangle \geqq\left\|P_{K} x-P_{K} x^{\prime}\right\|^{2}, \quad \forall x, x^{\prime} \in H,
$$

Received by the editors March 20, 1972.

AMS (MOS) subject classifications (1970). Primary 47H99; Secondary 46C05, $52 \mathrm{~A} 50$.

Key words and phrases. Hilbert space, convex set, projections.

(c) American Mathematical Society 1973 
whence it follows by the Schwarz inequality

$$
\left\|P_{K} x-P_{K} x^{\prime}\right\| \leqq\left\|x-x^{\prime}\right\|, \quad \forall x, x^{\prime} \in H .
$$

Thus, projections are nonexpansive mappings. Another simple consequence of (2) to be used in the sequel is

$$
P_{K}\left(P_{K^{-}} x+t\left(x-P_{K} x\right)\right)=P_{K} x, \quad \forall t \geqq 0, \forall x \in H .
$$

Should $K$ be a closed convex cone $C$ with vertex at 0 then (1) yields the equation

$$
\left\langle x-P_{C} x, P_{C} x\right\rangle=0, \quad \forall x \in H,
$$

obtained by replacing $y$ successively by 0 and $2 P_{C} x$.

Thus prepared we may now proceed to the statement and proof of our theorem:

THEOREM. The products, in either order, of two projections in Hilbert space on closed convex cones with vertices at the origin are both projections if and only if the given projections commute. In such a case the common value of the products is the projection on the intersection of the cones.

Proof. Necessity and the last part of the theorem result at once from the proposition

$$
\left\{P_{C_{1}} P_{C_{2}}=P_{C_{3}}\right\} \Rightarrow\left\{C_{3}=C_{1} \cap C_{2}\right\},
$$

whose proof runs as follows: Clearly $C_{3} \supset C_{1} \cap C_{2}$. On the other hand if $x \in C_{3}$ then by (6),

$$
\begin{aligned}
\left\|x-P_{C_{2}} x\right\|^{2} & =\left\langle x-P_{C_{2}} x, x\right\rangle \\
& =-\left\langle P_{C_{2}} x-P_{C_{1}} P_{C_{2}} x, P_{C_{1}} P_{C_{2}} x\right\rangle=0,
\end{aligned}
$$

that is, $x \in C_{2}$. Hence $C_{3} \subset C_{2}$, and since $C_{3} \subset C_{1}, C_{3} \subset C_{1} \cap C_{2}$. Thus $C_{1} \cap C_{2}=C_{3}$. To prove sufficiency it must be shown that if

$$
P_{C_{1}} P_{C_{2}}=P_{C_{2}} P_{C_{1}}
$$

then $P_{C_{1}} P_{C_{2}}=P_{C_{1} \cap C_{2}}$. Thus we assume (8) and write for any $x \in H, x_{1}=$ $P_{C_{1}} x, u_{1}=x-P_{C_{1}} x$. We then consider the points

$$
x(s, t)=t\left(x_{1}+s u_{1}\right)+(1-t) P_{C_{2}}\left(x_{1}+s u_{1}\right), \quad 0 \leqq s, t \leqq 1 .
$$

One checks first, with the assistance of (5) and (8), that

$$
\begin{aligned}
P_{C_{2}} P_{C_{1}} x(s, t) & =P_{C_{1}} P_{C_{2}} x(s, t)=P_{C_{1}} P_{C_{2}}\left(x_{1}+s u_{1}\right) \\
& =P_{C_{2}} P_{C_{1}}\left(x_{1}+s u_{1}\right)=P_{C_{2}} x_{1},
\end{aligned}
$$


and then, on use of (6), that

$$
\begin{aligned}
&\left\langle x(s, t)-P_{C_{1}} x(s, t), P_{C_{1}} P_{C_{2}} x(s, t)\right\rangle \\
&=\left\langle x(s, t)-P_{C_{1}} P_{C_{2}} x(s, t), P_{C_{1}} P_{C_{2}} x(s, t)\right\rangle \\
&-\left\langle P_{C_{1}} x(s, t)-P_{C_{2}} P_{C_{1}} x(s, t), P_{C_{2}} P_{C_{1}} x(s, t)\right\rangle \\
&= t\left\langle x_{1}-P_{C_{1}} P_{C_{2}} x(s, t), P_{C_{1}} P_{C_{2}} x(s, t)\right\rangle \\
&+(1-t)\left\langle P_{C_{2}}\left(x_{1}+s u_{1}\right)-P_{C_{1}} P_{C_{2}} x(s, t), P_{C_{1}} P_{C_{2}} x(s, t)\right\rangle \\
&+s t\left\langle u_{1}, P_{C_{2}} x_{1}\right\rangle \\
&= t\left\langle x_{1}-P_{C_{2}} x_{1}, P_{C_{2}} x_{1}\right\rangle \\
&+(1-t)\left\langle P_{C_{2}}\left(x_{1}+s u_{1}\right)-P_{C_{1}} P_{C_{2}}\left(x_{1}+s u_{1}\right), P_{C_{1}} P_{C_{2}}\left(x_{1}+s u_{1}\right)\right\rangle \\
&+s t\left\langle u_{1}, P_{C_{2}} x_{1}\right\rangle \\
&= s t\left\langle u_{1}, P_{C_{2}} x_{1}\right\rangle
\end{aligned}
$$

Moreover, since $P_{C_{2}} x_{1}=P_{C_{1}} P_{C_{2}} x_{1} \in C_{1}$,

$$
x(0, t)=\lim _{s \downarrow 0} x(s, t)=t x_{1}+(1-t) P_{C_{2}} x_{1} \in C_{1},
$$

and since projections are nonexpansive mappings (cf. (4)),

$$
\begin{aligned}
& \left\|s^{-1}\left(x(s, t)-P_{C_{1}} x(s, t)\right)\right\| \\
& \quad \leqq s^{-1}\|x(s, t)-x(0, t)\|=\left\|t u_{1}+(1-t) s^{-1}\left(P_{C_{2}}\left(x_{1}+s u_{1}\right)-P_{C_{2}} x_{1}\right)\right\| \\
& \quad \leqq t\left\|u_{1}\right\|+(1-t) s^{-1}\left\|P_{C_{2}}\left(x_{1}+s u_{1}\right)-P_{C_{2}} x_{1}\right\| \\
& \quad \leqq t\left\|u_{1}\right\|+(1-t)\left\|u_{1}\right\| \leqq\left\|u_{1}\right\| .
\end{aligned}
$$

Hence, $s^{-1}\left(x(s, t)-P_{C_{1}} x(s, t)\right)$ being bounded, it is possible to find, for any $t$ in the closed interval $[0,1]$, a positive sequence $s_{n} \downarrow 0$ such that $s_{n}^{-1}\left(x\left(s_{n}, t\right)-P_{C_{1}} x\left(s_{n}, t\right)\right)$ converge weakly to a limit $u_{t}$. From (10) one obtains

$$
\left\langle u_{t}, P_{C_{2}} x_{1}\right\rangle=t\left\langle u_{1}, P_{C_{2}} x_{1}\right\rangle, \quad 0 \leqq t \leqq 1 .
$$

Moreover, by (1),

$$
\left\langle s_{n}^{-1}\left(x\left(s_{n}, t\right)-P_{C_{1}} x\left(s_{n}, t\right)\right), P_{C_{1}} x\left(s_{n}, t\right)-y\right\rangle \geqq 0, \quad \forall y \in C_{1},
$$

whence letting $n \rightarrow \infty$,

$$
\left\langle u_{t}, x(0, t)-y\right\rangle \geqq 0, \quad \forall y \in C_{1} .
$$

In particular, replacing $y$ by $t x_{1}$ and by $t x_{1}+2(1-t) P_{C_{2}} x_{1}$, both belonging to $C_{1}$,

$$
(1-t)\left\langle u_{t}, P_{C_{2}} x_{1}\right\rangle \geqq 0, \quad-(1-t)\left\langle u_{t}, P_{C_{2}} x_{1}\right\rangle \geqq 0 .
$$


Now, assuming, as one may, that $0<t<1$, one derives from (13) and (11), $\left\langle u_{1}, P_{C_{2}} x_{1}\right\rangle=0$, that is

$$
\left\langle x-P_{C_{1}} x, P_{C_{2}} P_{C_{1}} x\right\rangle=0, \quad \forall x \in H .
$$

But then, since $P_{C_{1}}$ and $P_{C_{2}}$ commute,

$$
\begin{aligned}
\langle x- & \left.P_{C_{1}} P_{C_{2}} x, P_{C_{1}} P_{C_{2}} x-y\right\rangle \\
= & \left\langle x-P_{C_{1}} x, P_{C_{1}} P_{C_{2}} x-y\right\rangle+\left\langle P_{C_{1}} x-P_{C_{1}} P_{C_{2}} x, P_{C_{1}} P_{C_{2}} x-y\right\rangle \\
= & \left\langle x-P_{C_{1}} x,-y\right\rangle+\left\langle P_{C_{1}} x-P_{C_{2}} P_{C_{1}} x, P_{C_{2}} P_{C_{1}} x-y\right\rangle \\
= & -\left\langle x-P_{C_{1}} x, P_{C_{1}} x\right\rangle+\left\langle x-P_{C_{1}} x, P_{C_{1}} x-y\right\rangle \\
& +\left\langle P_{C_{1}} x-P_{C_{2}} P_{C_{1}} x, P_{C_{2}} P_{C_{1}} x-y\right\rangle,
\end{aligned}
$$

and by (1) and (6),

that is,

$$
\left\langle x-P_{C_{1}} P_{C_{2}} x, P_{C_{1}} P_{C_{2}} x-y\right\rangle \geqq 0, \quad \forall y \in C_{1} \cap C_{2},
$$

by (2). Q.E.D.

$$
P_{C_{1}} P_{C_{2}} x=P_{C_{1} \cap C_{2}} x, \quad \forall x \in H,
$$

\section{REFERENCE}

1. E. H. Zarantonello, Projections on convex sets in Hilbert space and spectral theory, Contributions to Nonlinear Functional Analysis, Academic Press, New York, 1971, pp. 237-424.

Centre de Recherches Mathématiques, Université de Montréal, Montréal, CANADA

Current address: Mathematics Research Center, University of Wisconsin, Madison, Wisconsin 53706 